\title{
Quelques paradoxes contemporains de la laïcité de l'État au Tchad
}

\section{Guy Bucumi}

\section{OpenEdition}

\section{$\checkmark$ Journals}

Édition électronique

URL : http://journals.openedition.org/rdr/437

DOI : $10.4000 /$ rdr.437

ISSN : 2534-7462

Éditeur

Presses universitaires de Strasbourg

\section{Édition imprimée}

Date de publication : 14 novembre 2019

Pagination : 155-173

ISBN : 979-10-344-0054-6

ISSN : 2493-8637

\section{Référence électronique}

Guy Bucumi, «Quelques paradoxes contemporains de la laïcité de l'État au Tchad », Revue du droit des religions [En ligne], 8 | 2019, mis en ligne le 25 novembre 2019, consulté le 19 novembre 2020. URL : http://journals.openedition.org/rdr/437 ; DOl : https://doi.org/10.4000/rdr.437

\section{(a) $(1)(9$}

La revue du droit des religions est mise à disposition selon les termes de la Creative Commons Attribution - Pas d'Utilisation Commerciale 4.0 International - CC BY-NC 4.0. 


\section{QUELQUES PARADOXESCONTEMPORAINS DELALAÏCITÉ DE L'ÉTAT AUTCHAD}

\section{Guy BUCUMI}

Université de Sherbrooke, Chaire de recherche Droit, Religion et Laïité

\section{Résumé}

La laïcité au Tchad, comme dans la plupart des pays francophones d'Afrique subsaharienne, s'est construite à partir du modèle français issu de la loi du 9 décembre 1905. La laïcité devint un principe constitutionnel depuis la première constitution issue du processus démocratique de 1993. Toutes les constitutions tchadiennes qui ont suivi affirment la séparation de l'État et des religions. L'introduction d'un serment confessionnel dans la nouvelle constitution adoptée le 4 mai 2018 suscite aujourd'hui beaucoup de débats dans le paysage religieux et politique du Tchad. Les controverses autour dudit serment interrogent la problématique de la laïcité consacrée par le constituant tchadien, son application et son adaptation dans la société tchadienne contemporaine.

\section{Abstract}

Secularism in Chad, like in most francophone countries in sub-Saharan Africa, was based on the French model resulting from the law of 9 December 1905. Secularism became a constitutional principle since the first constitution resulting from the democratic process in 1993. All Chadian constitutions that have followed have affirmed the separation of state and religions. The introduction of a religious oath in the new constitution, adopted on 4 May 2018, arouses much debate in Chad's religious and political fields. The controversies surrounding the oath are increasingly raising questions about the issue of secularism enshrined by the Chadian legislator, its application and adaptation in Chadian society today. 
$\mathrm{D}$ ans la dynamique des liens étroits, socio-politiques et historiques, unissant encore la France et ses anciennes colonies, le Tchad, à l'instar d'autres pays francophones d'Afrique noire, a adopté le modèle français de laïcité dans sa première constitution démocratique de 1993. La pratique de la laïcité au Tchad a toujours été, depuis lors, marquée par une volonté de séparation du temporel et du spirituel. En phase avec l'héritage colonial, l'adoption de la laïcité française par le constituant tchadien semblait correspondre pour le mieux aux attentes des dirigeants politiques de l'époque, soucieux de conduire le destin de leur peuple sur la base des institutions et principes constitutionnels avec lesquels ils avaient le plus de familiarité et de proximité.

Depuis sa constitutionnalisation, le principe de laïcité a été affecté par les mutations socio-politiques de ces trois dernières décennies. En effet, la construction du jeune État démocratique a très vite été confrontée à la résurgence du religieux dans le domaine public, qui a souvent caractérisé les différents régimes politiques depuis la I $^{\text {re }}$ République instaurée en 1993 après la conférence nationale souveraine ${ }^{1}$, dont l'organisation et la forme rappelaient les états généraux français de $1789^{2}$. Toutes les constitutions tchadiennes, depuis la conférence nationale souveraine de 1993, ont affirmé, sans ambiguïté, le caractère laïque de l'État et garantissent la liberté religieuse $^{3}$.

Le 30 avril 2018, le Tchad a adopté une nouvelle constitution par référendum, promulguée par le président de la République le 4 mai 2018. La nouvelle Loi fondamentale tournait ainsi une page de l'histoire constitutionnelle tchadienne en ouvrant une quatrième République que le président Idriss Deby Itno, au pouvoir depuis 1990, promettait innovante sur la question religieuse. La proposition d'une nouvelle République est apparue comme une réponse aux crises sociales qui se sont accrues ces dernières années. Dans un contexte sociopolitique morose et sous la pression d'un soulèvement populaire lié aux revendications sociales, le régime en place a proposé

1. Initiée pour la première fois au Bénin, la conférence nationale fut une assise populaire réunissant des délégués représentant toutes les couches de la population, toutes les régions, et y compris les Béninois de l'extérieur; ces assises mirent fin à la dictature et permirent une ouverture démocratique. Faisant figure de modèle de transition pacifique réussie, ce modèle béninois fut imité avec plus ou moins de succès par de nombreux États.

2. V. à ce propos R. Bujtenhuijs, La conférence nationale souveraine du Tchad. Un essai d'histoire immédiate, Paris, Karthala, 1993.

3. V. Constitution du 31 janvier 1996, Constitution du 6 juin 2005 et Constitution du 4 mai 2018. 
la tenue d'assises nationales, sur le modèle de la conférence nationale souveraine de 1993, afin de débattre, à bâtons rompus, de tous les maux qui minent la société tchadienne. Ce forum «sur la réforme des institutions» s'est tenu le 19 mars 2018 et a rassemblé plus de 800 personnes, représentant toutes les couches sociales du pays. La mission assignée à ces représentants était, principalement, d'examiner des propositions en vue d'ériger un "Tchad nouveau», doté de nouvelles institutions et de nouveaux mandats pour les hautes autorités du pays. Dans son discours inaugural, le chef de l'État a proposé aux participants de réfléchir notamment sur «la reconnaissance et la valorisation juridique de la morale religieuse comme l'une des caractéristiques des sociétés africaines et le reflet de l'identité nationale tchadienne ${ }^{4} »$. À l'issue de ces assises nationales, un projet de constitution fut proposé. C'est ce projet constitutionnel qui fut adopté sans difficulté par référendum et a ouvert la voie à une nouvelle République qui se veut en rupture avec les précédentes, notamment en matière de gouvernance et dans les rapports régissant les relations religions-État. En baisse de popularité et confronté à de sérieuses critiques sur sa gouvernance, le président Idriss Deby Itno a prôné un recours aux valeurs religieuses pour moraliser la vie publique et par là, renforcer la bonne gouvernance au Tchad ${ }^{5}$. Le chef de l'État, de confession musulmane, a, depuis quelques années, troqué son costume trois-pièces contre une djellaba et sa fréquentation assidue de la grande mosquée de N'Djamena, la capitale, pour la prière du vendredi, est de plus en plus remarquée.

Les débats sur la laïcité ont été ainsi ravivés avec l'introduction d'un serment confessionnel prévu par la nouvelle Loi fondamentale ${ }^{6}$. Ce serment imposé aux hauts fonctionnaires, allant des ministres aux directeurs des régies financières, en passant par les recteurs des universités publiques, est

4. Allocution d'ouverture du forum national inclusif au palais, 15 janv. 2019: https://www. presidence.td [consulté le 24 avr. 2019].

5. V. Constitution du 4 mai 2018, préambule, §§ 2 et 3 . Les articles 55 et 136 de la Constitution de 2018 instaurent quant à eux des règles de transparence et de bonne gouvernance auxquelles sont soumis tous les hauts fonctionnaires de l'État et mettent en place une Cour de répression des crimes économiques. Quant aux valeurs religieuses, la Constitution considère, dès le préambule, que «les valeurs morales et religieuses » constituent des valeurs fondamentales de la société tchadienne et concourent à la consolidation de l'unité du pays. L'article 36 dispose que l'État a le devoir de sauvegarder et de promouvoir les valeurs culturelles et religieuses du pays.

6. Art. $55 \& 2$ de la Constitution du Tchad: «[...] une catégorie de personnalités publiques et d'agents de l'État sont soumis à l'obligation de déclaration de patrimoine à la prise et à la fin de fonction et prêtent serment selon la formule confessionnelle consacrée par la loi »: http://www.laltdh.org/pdf/constution_tchad.pdf [consulté le 24 avr. 2019]. 
aujourd'hui décrié et contesté par les chrétiens essentiellement. Ce serment qui se prête devant la Cour suprême chargée d'en acter la régularité se fait également en présence du chef de l'État, garant de la Constitution, avant toute entrée en fonction.

Le refus d'une ministre de prêter ce serment confessionnel avant l'entrée en fonction du nouveau gouvernement, le 7 mai 2018, a largement contribué à alimenter le malaise actuel de la société tchadienne sur le plan religieux. Nommée à la tête du ministère de l'Aviation civile, des Transports et de la Météorologie, madame Rosine Djibergui, chrétienne évangélique, a refusé, devant le président de la Cour suprême mandaté pour valider les prestations de serment, de prêter ledit serment, en raison « de ses convictions religieuses, qui ne lui permettent pas de jurer au nom d'Allah ${ }^{7} »$. Limogée immédiatement, devant les caméras de la télévision nationale qui retransmettait en direct cette cérémonie de prestation de serment du nouveau gouvernement, elle a été remplacée sur-le-champ par le chef d'état-major particulier du président de la République ${ }^{8}$. L'histoire aurait pu s'arrêter là, mais cette scène a ravivé les débats sur la laïcité au Tchad et, plus généralement, toutes les questions liées à la liberté religieuse dans ce pays d'Afrique centrale. Il convient donc de faire une analyse de ces controverses, à travers une approche contextuelle de la laïcité au Tchad, en partant de ses fondements (1), avant de mettre en évidence les contradictions et les difficultés soulevées par ce serment confessionnel instauré par la Constitution de la IV République, actuellement en vigueur (2).

\section{LA LAÏCITÉ AU TCHAD}

Au Tchad, comme dans la plupart des pays africains aujourd'hui, la laïcité suscite des débats substantiels aux enjeux sociologiques, politiques et juridiques importants. Autrefois divisées entre paysans sédentaires au sud et nomades au nord, les ethnies du Tchad se définissent actuellement par leur appartenance confessionnelle ${ }^{9}$. Le pays est ainsi partagé géogra-

7. Explications de l'intéressée dans un journal local: https://tchadinfos.com/politique/tchaddjibergui-rosine-refuse-de-jurer-et-quitte-le-gouvernement/ [consulté le 24 avr. 2019].

8. V. le site officiel de la Présidence de la République du Tchad: https://www.presidence.td/ fr-news-3214.html [consulté le 24 avr. 2019].

9. Dans le dernier recensement de l'Institut national de la statistique, des études économiques et démographiques du Tchad datant de 2009, le Tchad compte $58 \%$ de musulmans, $18 \%$ de catholiques, $16 \%$ de protestants et les $8 \%$ restant pratiquent des religions autochtones: http://www.inseed-td.net/index.php/thematiques/statistique-demographique/ population [consulté le 29 mai 2019]. 
phiquement par une frontière religieuse, avec un nord majoritairement musulman et un sud essentiellement chrétien. Cette «menaçante frontière religieuse ${ }^{10} »$ est, selon certains analystes, une bombe à retardement pour la stabilité de ce pays et de toute la région d'Afrique centrale. La population tchadienne est aujourd'hui marquée par ce clivage, cette fracture nord-sud au parfum religieux qui sert de distinction et caractérise depuis quelques décennies ces régions historiquement antagonistes.

L'histoire religieuse du Tchad s'est construite tantôt sur une collaboration, tantôt sur une ignorance entre les sphères temporelle et spirituelle. L'administration coloniale française a tenu, tout au long de sa présence, une position ambivalente vis-à-vis de l'islam qui fut considéré comme un moyen d'approche de la société tchadienne dont il constituait le socle. Même si l'État colonial n'a jamais caché sa méfiance vis-à-vis de cette religion, la première à s'implanter dans la région d'Afrique centrale, il n'a jamais pris de mesures visant à l'interdire ni même à freiner son expansion. L'exception fut faite aux régions frontalières du Cameroun et de la Centrafrique où l'islam aurait perturbé l'implantation des missions catholiques. C'est paradoxalement durant la période coloniale que l'islam a effectué une percée significative dans la société tchadienne, notamment dans les régions du nord du pays. Mais l'administration coloniale ne fut jamais totalement neutre à son égard en instaurant non pas une séparation, mais une surveillance et un contrôle accrus. Dans tout l'empire colonial français plus globalement, l'islam constitua une exception notoire à la loi de séparation des Églises et de l'État de $1905^{11}$. Le contrôle des populations, à travers le contrôle de la religion musulmane, devint l'une des clés de la politique religieuse de la France au Tchad ainsi que dans d'autres territoires majoritairement musulmans. En effet, l'administration coloniale n'oubliait pas que l'islam n'était pas seulement une religion, mais aussi «une civilisation, comme la civilisation chrétienne et qu'il serait imprudent de traiter ces deux aspects séparément ${ }^{12}{ }$. Selon les lieux et les circonstances donc, la France coloniale a, durant un siècle et demi de colonisation, traité, combattu et encadré la religion musulmane. Après l'indépendance, au début des années 1960, il y a eu une soudaine montée en puissance des forces politiques se réclamant de l'islam.

10. Titre d'un reportage spécial du journal Libération sur les religions au Tchad: S. SMith, «Tchad, la menaçante frontière religieuse», Libération, 30 janv. 1995.

11. En effet, la loi du 9 décembre 1905 disposait en son article 43 \& 2: «Des décrets en Conseil d'État détermineront les conditions dans lesquelles la présente loi sera applicable en Algérie et aux colonies. »

12. F. Braudel, L’identité de la France, Paris, Flammarion, t. 2, 1986, p. 169. 
Le Tchad, du fait de sa situation géographique, a toujours été considéré comme le lieu d'un rapport de force entre la poussée de l'islam et les résistances chrétiennes du reste de l'Afrique équatoriale française (AEF) ${ }^{13}$. Dès la création des premiers partis politiques en 1947, ceux d'obédience musulmane bénéficièrent de soutiens financiers et matériels, en provenance du Moyen-Orient essentiellement, pour leur conquête du pouvoir et une implantation solide. Pourtant, les rênes du pouvoir furent très tôt confiées par l'administration coloniale aux hommes politiques issus de la minorité chrétienne: d'abord François Tombalbaye puis Félix Mallum qui dirigèrent le Tchad jusqu'en 1979. On ne saurait comprendre l'évolution de la politique religieuse de ce pays sans considérer, sur le plan législatif, les différentes lois en la matière, qui ont toujours servi de baromètre dans les relations entre les deux sphères, profane et sacrée. L'encadrement du champ religieux au Tchad est passé par différentes législations religieuses sur lesquelles s'est fondée la gestion cultuelle depuis l'époque coloniale. Il convient dès lors d'évoquer l'histoire de la laïcité au Tchad à travers les différents principes constitutionnels et leur évolution dans le temps, d'une part (1.1), et d'autre part d'analyser les réactions des confessions religieuses au nouveau texte constitutionnel qui fait polémique aujourd'hui (1.2).

\subsection{LES PRINCIPES CONSTITUTIONNELS EN MATIÈRE RELIGIEUSE}

Au Tchad, comme ailleurs en Afrique, le statut légal des cultes reflète toujours l'état des rapports entre les deux pouvoirs, spirituel et temporel. La collaboration s'imposait généralement sous le régime colonial, car l'Administration ne pouvait se passer de la religion musulmane, solidement implantée dans la société tchadienne bien avant le début de l'empire colonial.

Sur le plan législatif, ces relations furent dès le début de la colonisation régies par la loi sur les associations du $1^{\text {er }}$ juillet 1901. Après l'adoption de la loi de 1905 sur la séparation des Églises et de l'État, le régime colonial des cultes fut ensuite principalement régi par les décrets Mandel du 16 janvier 1939 et du 6 décembre 1939.

Par la suite, les décrets Jacquinot du 14 septembre 1951 ont réglé la question matrimoniale et ont, sur ce point, opéré un profond bouleversement de la

13. En Afrique subsaharienne, l'Empire colonial français était divisé en deux entités, l'une au centre et l'autre à l'Ouest. Il y avait ainsi l'Afrique équatoriale française (AEF) regroupant tous les territoires d'Afrique centrale et l'Afrique occidentale Française (AOF) pour les territoires de l'Ouest. Chacun de ces regroupements avait à sa tête un gouverneur général. 
loi islamique applicable en la matière. Ces décrets, qui furent perçus comme des dispositions anti-musulmanes, énonçaient par exemple que «chacun des futurs époux, même mineur, doit consentir personnellement ${ }^{14} »$. L'objectif principal était de favoriser l'émancipation de la femme dans les relations entre époux. Ces décrets restèrent applicables tout au long de la période coloniale.

La première législation en matière religieuse du jeune État fut une ordonnance prise au lendemain de l'indépendance. Afin de marquer la rupture avec les décrets Mandel et les décrets Jacquinot qui régissaient jusque-là les relations religions-État dans les colonies, l'ordonnance prise en 1962 par les nouveaux dirigeants disposait que «la loi du $1^{\text {er }}$ juillet 1901 sur les associations ainsi que les textes qui l'ont complétée et modifiée, sont abrogés ${ }^{15} »$. Cette ordonnance instituait un régime d'autorisation pour toute association désirant s'implanter au Tchad, y compris pour les congrégations religieuses ainsi que les associations à caractère religieux. On restait, en matière de gestion cultuelle, dans l'esprit de la loi de 1901, tout en y ajoutant des dispositions propres au nouvel État indépendant. Ces associations devaient adresser une demande d'autorisation «au ministre de l'intérieur qui se prononce sur l'autorisation ou le refus d'installation d'une association religieuse ${ }^{16} »$; les autorités administratives ou juridiques "peuvent procéder au contrôle de régularité des associations à chaque fois que le besoin se fait sentir ${ }^{17} »$. On note ici une volonté de contrôle strict de l'appareil étatique sur les associations religieuses.

C'est cette ordonnance qui est restée en vigueur pendant plusieurs décennies, la législation en matière religieuse n'étant devenue une préoccupation majeure des pouvoirs publics qu'avec la montée en puissance d'un islamisme radical autour du lac Tchad. En juin 2015 par exemple, après deux attentats terroristes attribués à la secte islamiste nigériane Boko Haram au centre de la capitale N'Djamena, le Premier ministre a interdit par arrêté tout port de la burqa et a demandé à toutes les autorités religieuses islamiques de sensibiliser les fidèles musulmans à cette mesure ${ }^{18}$. Une nouvelle ordonnance et un décret ont été pris dans la foulée pour réglementer successivement les

14. Art. $108, \S 2$.

15. Ord. 62-27, 28 juill. 1962, art. 15.

16. Ibid., art. $5 \S 2$.

17. Ibid., art. $5 \& 6$.

18. Arrêté no 009/PR/PM/MSPI/SG/2016. 
réunions publiques ${ }^{19}$ et les manifestations sur la voie publique ${ }^{20}$. Les autorités tchadiennes ont régulièrement invoqué ces deux dernières réglementations pour interdire les rassemblements religieux et les processions. En effet, l'ordonnance $n^{\circ} 45 / 62$ prévoit que les réunions publiques «ne peuvent avoir lieu sans autorisation préalable» (art. 4) et le décret dispose que «tout cortège, défilé, sortie sur la voie publique est soumis à l'obligation d'une déclaration préalable et à l'obtention d'une autorisation sauf les sorties conformes aux usages locaux» (art. 8). Cette dernière formule a souvent permis aux autorités locales de décider, de façon discrétionnaire, à quelle confession religieuse octroyer une autorisation, en invoquant sa conformité «aux usages locaux». L'ancien envoyé spécial des Nations unies sur le droit de réunion pacifique et d'association, Maina Kiai, avait d'ailleurs dans son rapport fustigé ladite formule, estimant que cette exigence d'une autorisation «fait de la liberté religieuse au Tchad, un privilège» et que le but ultime d'une notification aux autorités devrait simplement «permettre aux autorités de faciliter l'exercice du droit à la liberté religieuse et de réunion, de manière pacifique ${ }^{21} »$. Le rapporteur spécial des Nations unies a également insisté sur le fait que «même les notifications aux autorités ne devraient concerner que les grands rassemblements présentant des risques de trouble à l'ordre public ${ }^{22} »$.

Sur le plan constitutionnel, le Tchad a, depuis la première constitution post-indépendance, affirmé «solennellement la volonté du peuple tchadien, de vivre ensemble dans le respect de sa diversité religieuse $[\ldots]^{23}$ » et déclaré son attachement aux «principes de droits de l'homme tels que définis par la Charte des Nations unies de 1945 et la Déclaration universelle des droits de l'homme de $1948^{24} »$.

Cette première constitution post-indépendance et celle qui l'a suivie en 1973 étaient en revanche, toutes deux muettes sur le principe de laïcité. C'est la Constitution de 1982 qui consacre, pour la première fois la laïcité

19. V. Ord. $n^{\circ} 45 / 62$.

20. V. D. n ${ }^{\circ} 193 / 620$.

21. Pour le rapport, V. https://www.ohchr.org/Documents/HRBodies/HRCouncil/RegularSession/Session20/A-HRC-20-27_fr.pdf. S'appuyant sur ces recommandations de Maina Kiai, l'atteinte à la liberté religieuse au Tchad a également fait l'objet d'un rapport d'Amnesty International en octobre 2018: Tchad. Analyse juridique de l'ordonnance portant régime des associations: https://www.amnesty.org/download/Documents/AFR2092402018FRENCH. PDF [consultés le 24 avril 2019].

22. Ibid.

23. L. constit. $\mathrm{n}^{\circ}$ 02-62, 2 avr. 1962, préambule, $\S 1$.

24. Ibid., \& 2 . 
en affirmant le «caractère laïque de l'État tchadien ${ }^{25} »$. Après avoir réaffirmé le caractère laïque de l'État dans les mêmes termes que la précédente, la Loi fondamentale de 1996 issue du processus démocratique a quant à elle précisé que «la République du Tchad se fonde sur les principes de démocratie, du règne de la loi et de la justice en affirmant la séparation des religions et de l'État ${ }^{26}{ }$. Cette même Constitution de 1996 rappelait la valeur constitutionnelle des divers engagements internationaux du Tchad en matière religieuse, comme le Pacte international relatif aux droits civils et politiques ratifié en 1995 qui dispose en son article 18 que «Toute personne a droit à la liberté de pensée, de conscience et de religion; ce droit implique la liberté d'avoir ou d'adopter une religion ou une conviction de son choix, ainsi que la liberté de manifester sa religion ou sa conviction, individuellement ou en commun, tant en public qu'en privé, par le culte et l'accomplissement des rites, les pratiques et l'enseignement». La question du droit de changer de religion, qui fut l'objet de réserves de plusieurs pays musulmans, ne s'est pas posée dans le cas du Tchad. En revanche, celui-ci n'a pas adhéré au protocole facultatif sur la compétence du Comité des droits de l'homme. À la fin de l'année 1994, le Tchad a toutefois créé une Commission nationale des droits de l'homme (CNDH) dont le rôle était de «formuler des avis sur les questions des droits de l'homme à l'intention du gouvernement ${ }^{27}$ » et de «participer à la révision et à la rédaction des lois pour s'assurer de leur conformité avec la Charte des droits de l'homme issue de la conférence nationale, ainsi qu'avec les traités régionaux et internationaux relatifs aux droits de l'homme ${ }^{28} »$. Hébergée dans les bureaux de la primature qui finance ses activités et met du personnel à sa disposition, la commission a brillé par son manque d'indépendance, d'autonomie et de crédibilité. Trois décennies plus tard, son bilan et ses activités sont de plus en plus critiqués ${ }^{29}$.

Le Tchad étant membre de l'Union africaine, le texte de 1996 réaffirmait également son attachement à la Charte africaine des droits de l'homme et des peuples, adoptée en 1981, un texte qui garantit «la liberté de conscience, la profession et la pratique libre de la religion ${ }^{30} »$.

25. Constitution de 1982, art. $1^{\text {er }}$.

26. Constitution du 31 mars 1996 révisée, art. $1^{\text {er }}$, al. 1 et 2.

27. L. $n^{\circ}$ 94-031, 9 sept. 1994 , art. 3 (a).

28. Ibid., art. 3 (b).

29. V. à ce propos les critiques du Représentant de la Commission des droits de l'homme de l'ONU, in E. Aouj, Rapport sur la situation des droits de l'homme au Tchad, déc. 1998, E/CN.4/1998/R.3.

30. Charte africaine des droits de l'homme et des peuples de 1981 (entrée en vigueur en 1986), art. 8 . 
Enfin, la récente constitution tchadienne adoptée en mai 2018, tout en reprenant la consécration du principe de laïcité dans les mêmes termes que les deux précédentes lois fondamentales, proclame, dès le préambule, que «la tolérance politique, ethnique et religieuse, le pardon, le dialogue interreligieux constituent des valeurs fondamentales concourant à la consolidation de l'unité et de la cohésion nationales ${ }^{31} »$. Elle réaffirme en outre, toujours dans son préambule, son attachement aux différents instruments internationaux en matière de droits humains ratifiés par le Tchad. Cette Constitution de mai 2018 rappelle la religiosité des sociétés tchadiennes et affirme une volonté de «bâtir un État fondé sur des valeurs africaines $[\ldots]^{32} »$. Du fait de la forte religiosité des sociétés africaines, les valeurs africaines trouvent leurs fondements dans les préceptes religieux.

Enfin, ce même texte introduit, pour la première fois dans l'histoire constitutionnelle du Tchad, un serment confessionnel qui fera l'objet d'une analyse approfondie dans la seconde partie de cet article. L'allusion claire faite à l'islam dans la formulation de ce serment paraît nettement paradoxale avec le caractère laïque consacré par la même Loi fondamentale. Ce nouveau rôle prépondérant de l'islam dans la sphère publique ne peut qu'inquiéter les autres obédiences religieuses qui ont accueilli, avec des nuances, les récentes évolutions des relations religions-État dans le pays et s'inquiètent désormais de ce glissement de l'État vers une compromission en faveur de la religion musulmane majoritaire.

Le Tchad s'est doté d'un Conseil constitutionnel depuis la Loi fondamentale du 31 mars $1996^{33}$, mis en place par la loi organique de $1998^{34}$. Cette institution est indépendante selon la loi organique qui régit son organisation, mais connaît des difficultés quant à l'exercice de cette indépendance. En effet, le budget de fonctionnement du Conseil constitutionnel est soumis au président de la République qui doit l'approuver ${ }^{35}$. Deux remarques s'imposent par ailleurs quant aux procédures de fonctionnement dudit conseil depuis sa création: l'une est relative à sa saisine et l'autre à la mise en application de ses décisions. On peut relever tout d'abord l'absence d'autosaisine et la limitation des catégories de personnes habilitées à saisir le Conseil constitutionnel.

31. Constitution de la République du Tchad du 4 mai 2018, préambule.

32. Ibid., préambule, \& 1 .

33. Constitution tchadienne de 1996, Titre VII : «Du Conseil constitutionnel».

34. L. organique $\mathrm{n}^{\circ}$ 019/PR/98, 2 nov. 1998, portant organisation et fonctionnement du Conseil constitutionnel.

35. L. organique $n^{\circ}$ 019/PR/98, art. 38 . 
En effet, ne peuvent le saisir que le président de la République, le Premier ministre, le président de l'Assemblée nationale ou un dixième des membres de l'Assemblée nationale pour statuer sur la constitutionnalité d'une loi ${ }^{36}$. Ensuite, bien que les décisions dudit conseil «ne soient susceptibles d'aucuns recours» et qu'elles «s'imposent aux pouvoirs publics et à toutes les autorités administratives militaires et juridictionnelles ${ }^{37}$ », rien n'est prévu dans le cas où les autorités refusent de se soumettre aux décisions du Conseil constitutionnel. Il n'est pas rare que les décisions de la haute juridiction constitutionnelle ne soient pas suivies d'effet; le cas le plus récent étant la décision $\mathrm{n}^{\circ}$ 002/CC/SG/06 du 17 février 2006 sur la loi constitutionnelle portant prorogation de la législature alors en cours. Pour le reste, la procédure devant le Conseil constitutionnel est écrite et non contradictoire. Les séances ne sont pas publiques et les intéressés ne peuvent pas demander à y être entendus. Toutes ces raisons expliquent pourquoi la haute juridiction tchadienne en matière constitutionnelle n'a pas été jusqu'à présent saisie pour se prononcer sur la nouvelle Loi fondamentale.

\subsection{LES RÉACTIONS DES CONFESSIONS RELIGIEUSES AU SERMENT CONFESSIONNEL}

Le Tchad étant essentiellement musulman et catholique, nous n'envisageons pas d'étudier toutes les religions, mais analyserons essentiellement les attitudes de ces deux confessions par rapport à la laïcité.

Face à l'islamisation de la classe politique tchadienne, l'Église catholique a recouru à la technique concordataire dans ses rapports avec l'État, par le biais d'un accord-cadre dont il convient d'examiner les caractéristiques et le contenu.

Si chaque époque est porteuse de modèles de relations religions-État, le modèle choisi par le Saint-Siège avec la plupart des pays d'Afrique centrale est tributaire d'exigences tant politiques que sociétales. Le recours à la traditionnelle technique concordataire est apparu comme courant au $\mathrm{xxI}^{\mathrm{e}}$ siècle en Afrique. L'explosion des concordats africains est alors surtout le résultat d'un fort déclin de l'Église catholique, dû à l'adhésion de plus en plus massive de fidèles catholiques aux nouveaux mouvements religieux et à la percée de l'islam rencontrant les faveurs des autorités politiques. C'est le second phénomène qui est particulièrement visible au Tchad. Les concordats sont 
pour l'Église catholique romaine l'occasion de réaffirmer la présence ecclésiastique et ecclésiale. Dans le cas du Tchad, le concordat signé en 2013 vise à transposer dans le droit local certaines dispositions canoniques du fait du contexte particulier du phénomène religieux dans la région, marquée par les différents conflits religieux recensés encore aujourd'hui. Ce concordat vise également à contourner les législations religieuses étatiques de plus en plus exigeantes et restrictives à l'égard des associations religieuses minoritaires.

L'accord-cadre signé le 6 novembre $2013^{38}$ entre l'État tchadien et le Saint-Siège rappelle dans son préambule la contribution de l'Église catholique «au développement spirituel, social, éducatif et culturel du peuple tchadien $^{39} »$. Tout en reconnaissant le «caractère laïque de l'État», les deux parties assurent se référer dans leur coopération à « tous les principes internationalement reconnus en matière de liberté religieuse ${ }^{40} »$. Dans ce nouveau cadre juridique, l'État tchadien garantit aux fidèles catholiques la «liberté de professer publiquement leur foi ${ }^{41}$ » et à l'Église catholique «la liberté de s'organiser selon le droit canonique, d'accomplir sa mission pastorale et d'exercer le culte ${ }^{42}$ ». La République tchadienne garantit aussi «à l'Église catholique, tant à ses fidèles en général, ainsi qu'aux responsables et aux membres des institutions ecclésiastiques, la liberté de communiquer et de se maintenir en relation avec le Saint-Siège, avec les conférences épiscopales d'autres pays, tout comme avec les personnes et les organismes présents à l'intérieur et à l'extérieur du pays ${ }^{43} »$.

Si le contenu et la typologie des concordats ont aujourd'hui évolué, l'esprit en est également modifié. En effet, ces concordats avec les États africains comme le Tchad, dénommés «accords-cadres», garantissent, dans la plupart des cas, une forme de laïcité, avec une attention marquée et revendiquée de part et d'autre au droit à la liberté religieuse. L'accord-cadre avec le Tchad fixe essentiellement le cadre juridique entre les deux institutions et la reconnaissance par l'État de l'Église catholique, ainsi que de toutes les institutions ecclésiastiques catholiques présentes sur son sol. Dans le nouveau contexte des relations religions-État, cet accord-cadre permet de garantir la liberté religieuse comme un droit fondamental constitutionnellement consacré. Cette «politique

38. V. http://bibliotecanonica.net/docsap/btcapc.pdf [consulté le 24 avr. 2019].

39. Accord-cadre, préambule, \& 4.

40. Ibid., \& 2 .

41. Accord-cadre, art. $3 \S 1^{\mathrm{er}}$.

42. Ibid., art. $3 \& 2$.

43. Art. $3 \S 4$. 
d'accommodement» de l'Église lui assure une cohabitation apaisée avec les États africains majoritairement musulmans. En effet, l'Église tchadienne a toujours su maintenir un compromis avec les différents régimes qui se sont succédé et de ce fait n'a jamais été perçue comme une menace pour l'État. Si l'Église catholique a toujours entretenu d'excellents rapports avec l'État tchadien, elle en a fait de même avec la communauté musulmane. Entre ces deux confessions religieuses, il existe depuis longtemps une « union sacrée » des leaders religieux, lesquels se rencontrent régulièrement. Ils ont souvent mené conjointement des activités, telles que des médiations dans le cadre des différentes crises sociales qui ont secoué le Tchad ces dernières années.

L'introduction du serment confessionnel dans la nouvelle constitution est considérée par l'Église catholique et d'autres groupements religieux comme une officialisation de l'islam qui devient ainsi de facto une religion d'État. Face à ce qu'ils vivent comme une injustice, les responsables de ces diverses confessions religieuses demandent aujourd'hui la réinstauration d'un État laïque, garant de l'équité entre toutes les confessions religieuses légalement établies, conformément aux constitutions antérieures.

L'Église catholique, par la voix de $\mathrm{M}^{\mathrm{gr}}$ Edmond Djitangar, évêque de N'Djamena, déplore les limogeages de plusieurs responsables politiques essentiellement catholiques ayant refusé de prêter ce serment «au nom d'Allah» et parle d'une véritable persécution religieuse en cours et donc d'une violation des libertés fondamentales. Dans une lettre adressée au président de la République, la conférence épiscopale du Tchad a demandé l'abrogation du serment, en dénonçant son caractère discriminatoire et la violation flagrante du principe constitutionnel de laïcité.

Les protestants de leur côté, par le biais de l'Entente des Églises et missions évangéliques au Tchad regroupant la quasi-totalité des Églises protestantes, déplorent l'atteinte aux principes constitutionnels qui fondent la République, dont «la laïcité et la cohésion nationale ${ }^{44} »$. Face aux critiques des responsables chrétiens, le président Deby avait tenu à rassurer la communauté chrétienne quant à l'introduction du serment confessionnel. Dans une récente conférence de presse, il assurait «appliquer simplement la volonté du peuple $^{45}$ » et affirmait que l'objectif n'est pas de porter atteinte à la laïcité de

44. «Tchad, le débat sur la laïcité rebondit » : http://www.rfi.fr/afrique/20180731-tchad-debatlaicite-rebondit [consulté le 24 avr. 2019].

45. Conférence de presse du Président Deby, le 12 août 2018: http://www.rfi.fr/ afrique/20180812-tchad-celebration-independance-58-annniversaire-greve-laicite-discoursdeby [consulté le 24 avr. 2019]. 
l'État, mais d'« empêcher les détournements et la corruption qui gangrènent la fonction publique» en passant par un rappel des valeurs religieuses «qui ont toujours caractérisé les sociétés tchadiennes ${ }^{46} »$. Catholiques et protestants reprochent à l'État de ne pas protéger les religions minoritaires par rapport à l'islam, religion pratiquée par la grande majorité des Tchadiens, qui se trouve aujourd'hui renforcée de facto par ce serment confessionnel dont la formulation a été confiée au Conseil islamique du Tchad.

L'environnement politique actuel ne favorise guère en effet le plein épanouissement des autres confessions religieuses qui semblent subir une pression de l'islam. Cette récente montée en puissance des communautés islamiques au Tchad est aujourd'hui révélatrice d'un principe de laïcité dont l'application a toujours été confuse. En effet, bien que consacrée par les constitutions successives depuis 1973, la séparation des pouvoirs politique et religieux fut combattue et diabolisée par les responsables musulmans qui ont toujours feint d'ignorer cette distinction, rendant donc malléable la frontière poreuse entre les deux sphères. La visibilité de l'islam a souvent servi de baromètre au principe de laïcité, provoquant d'incessantes controverses. Cette problématique soulève enfin l'éternelle question de la compatibilité du principe de laïcité avec l'islam, un principe dont l'application est aujourd'hui mise à l'épreuve par le serment confessionnel (2).

\section{LE SERMENT CONFESSIONNEL}

L'application de la laïcité au Tchad se trouve confrontée à diverses remises en cause. Perçue et comprise sous des schémas strictement différents, elle induit des luttes et crée aujourd'hui des tensions non seulement entre confessions religieuses, mais aussi entre ces dernières et l'État. Le cœur des débats actuels est le serment confessionnel introduit dans le nouveau texte constitutionnel dont il convient d'apprécier les motivations avérées et non avérées (2.1) ainsi que son contenu et la charte déontologique sur laquelle il s'appuie (2.2).

\subsection{LES MOTIFS DE L'INTRODUCTION DU SERMENT CONFESSIONNEL}

À l'origine de cette disposition constitutionnelle qui fait polémique aujourd'hui, on trouve un pays gangréné par une mauvaise gouvernance, des 
crises sociales à répétition et un pouvoir qui vacille. L'évolution du contexte socio-politique a fait émerger une dynamique associative, favorisant une multitude d'organisations islamiques dont plus de la moitié sont localisées dans la capitale N'Djamena. Structurées et disposant d'un soutien financier, essentiellement en provenance du Moyen-Orient, elles ont bénéficié d'une large adhésion populaire et leur influence n'a fait que croître. Le régime tchadien a opté pour l'institutionnalisation de cette dynamique perçue comme une menace, en créant un Conseil supérieur des affaires islamiques (CSAI). Les associations islamiques sont ainsi devenues des acteurs incontournables de la scène politique tchadienne. Leur efficacité et leur influence tiennent à leur capacité de mobilisation des fidèles musulmans qui constituent un électorat sans lequel on ne peut accéder au pouvoir. Si elles sont officiellement apolitiques, leur engagement, même inavoué, dans des luttes politiques est de plus en plus évident. Face au discrédit de l'État, accentué par la difficile résorption des crises sociales au sein des classes populaires, elles ont joué le rôle de porte-parole des mouvements de mécontentement qui ont secoué le Tchad et menacé le pouvoir du président Idriss Deby Itno. En effet, les conditions de vie insatisfaisantes des populations les ont souvent poussées à se réfugier auprès des associations religieuses islamiques, de plus en plus souvent financées par des fonds en provenance des Émirats arabes unis notamment. Ces associations se sont ainsi substituées à l'État défaillant dans les domaines telles la santé et l'éducation ${ }^{47}$, apparaissant ainsi comme « une alternative sociale crédible ${ }^{48} »$.

Dans les propositions de sortie de crise, le chef de l'État tchadien a opté pour une coopération renforcée avec les chefs religieux musulmans qu'il a reçus en audience, peu avant de convoquer le forum inclusif de toutes les forces vives de la nation. Bien que la coopération entre le politique et le religieux ne date pas d'aujourd'hui, c'est la première fois que les pouvoirs publics utilisent l'islam comme symbole d'unité nationale, en cherchant à s'appuyer sur les différentes confréries pour associer islamisation et étatisation. Pour pallier la mauvaise gouvernance de la manne pétrolière, principal reproche fait au régime, le président Deby décida d'y associer les leaders religieux par

47. La capitale N'Djamena compte à elle seule plus de 50 instituts et écoles coraniques. Afin de contrer cette influence, l'État a inauguré le 30 janvier 2018 un Centre pour le développement et la prévention de l'extrémisme violent (CEDPE) : https://www.la-croix.com/ Religion/Islam/Le-Tchad-ouvre-centre-deradicalisation-2018-01-31-1200910232 [consulté le 24 avr. 2019].

48. Propos d'un prêtre catholique sur la montée de l'islamisme au Tchad: https://www.lacroix.com/Religion/Islam/Le-Tchad-ouvre-centre-deradicalisation-2018-01-31-1200910232 [consulté le 24 avril 2019]. 
la loi $\mathrm{n}^{\circ}$ 002/PR/06 ${ }^{49}$ portant gestion des revenus pétroliers au Tchad. Outre les dispositions concernant la répartition des revenus pétroliers, cette loi prévoit en son article 16 la création et la composition de l'organe chargé de la surveillance, dénommé «Collège de contrôle et de surveillance des revenus pétroliers ». Parmi les membres, et c'est une nouveauté, figure un représentant des confessions religieuses. Même si aucune précision n'est donnée sur les modalités de choix de ce représentant dans ledit collège de gestion, la désignation répétitive d'un membre issu de la communauté musulmane à ce poste révèle bien les attentes et les objectifs du régime en place qui désire assurer sa longévité aux affaires. Le Tchad a été longtemps présenté comme un pays "musulman et laiqque», parce que bien que l'immense majorité des Tchadiens se réclamaient de l'islam, il n'existait pas de religion d'État. En effet, l'islam et l'État représentaient jusque-là dans le paysage tchadien deux entités officiellement distinctes. Avec l'actuelle Loi fondamentale, la place de l'islam fait aujourd'hui l'objet d'une profonde mutation et le discours religieux est entré officiellement sur la scène politique. Le bouleversement le plus notable est actuellement lié au serment confessionnel dont le contenu soulève d'importantes ambiguïtés (2.2).

\subsection{LA FORMULE DU SERMENT ET LA CHARTE DÉONTOLOGIQUE}

La nouvelle constitution tchadienne adoptée en mai 2018 dispose en son

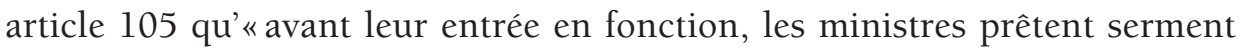
devant le président de la République, suivant la formule confessionnelle consacrée par la loi ${ }^{50} »$. L'ordonnance $n^{\circ}$ 013/PR/2018 précise quant à elle le contenu de ce serment. Ainsi, toute personne nommée à de hautes fonctions étatiques doit prêter serment «au nom d'Allah, le Tout Puissant, d'être loyal dans son travail, de ne pas trahir la nation et le peuple, de ne pas détourner les deniers publics, de ne pas dévoiler les secrets de l'État et de respecter les engagements contenus dans une charte déontologique ${ }^{51} »$. La charte déontologique dont il est question énonce une dizaine de valeurs que doivent défendre tous les hauts cadres de l'État. Ces valeurs vont du «sens de l'État et l'amour de la patrie» à «la civilité, la courtoisie et la modestie», en passant par la bonne gouvernance, la responsabilité, l'intégrité et la probité. Ces valeurs doivent prévaloir sur toute "particularité régionale, ethnique,

49. Cette nouvelle loi a amendé la loi $n^{\circ}$ 001/PR/99 du 11 janvier 1999 régissant la gestion des revenus pétroliers au Tchad.

50. Constitution de la République du Tchad, art. $105 \& 2$.

51. D. présidentiel nº 013/PR/2018, 24 mai 2018. 
politique et religieuse ${ }^{52} »$. Le libellé du serment confessionnel, obligatoire depuis mai 2018 pour accéder à une haute fonction étatique, est aujourd'hui contesté par les confessions chrétiennes qui le jugent inconstitutionnel et discriminatoire. De fait, imposer un serment confessionnel dans une République qui se dit laïque semble revenir à imposer une croyance religieuse à toute la Nation et constitue donc une atteinte à la liberté de croyance et de religion, garantie pourtant par la même Loi fondamentale adoptée le 4 mai 2018 ainsi que par les divers instruments internationaux ratifiés par le Tchad. Lorsqu'en outre le contenu de ce même serment fait allusion à une confession religieuse donnée, cela revient à privilégier clairement une confession religieuse au détriment des autres et à porter atteinte à la neutralité de l'État tchadien. Par ce serment confessionnel, l'État tchadien tend d'une part à reconnaître le fait religieux comme l'une des valeurs spécifiques de la société, et manifeste d'autre part la volonté de l'assumer dans ses institutions. Mais l'imposer met à mal le principe de laïcité consacré par la Constitution qui prône, sans aucune ambiguité, une séparation stricte de l'État et des religions. On constate aujourd'hui un jeu d'appropriation réciproque des symboles de l'État et de la confession musulmane: le pouvoir politique prend une essence divine et donne une légitimité religieuse musulmane aux hauts fonctionnaires de l'État qu'il veut soumettre au serment. Cette situation inédite au Tchad soulève la question des répercussions politiques d'une telle entreprise, dans un pays où les pouvoirs politique et religieux étaient déjà étroitement liés et où l'un et l'autre cherchaient à se contrôler mutuellement.

Au-delà de la question de la laicité, on voit dans cette démarche des autorités tchadiennes un nouveau mode de gestion cultuelle qui est, on peut le penser, tributaire d'un contexte régional de plus en plus tendu dans le domaine religieux. En effet, le Tchad est frontalier du Nigéria dans sa partie nord et ouest, pays où sévit la secte islamiste Boko Haram qui s'implante progressivement dans tous les pays situés autour du bassin du lac Tchad. Au sud se trouve la République centrafricaine, confrontée depuis 2013 à une guerre religieuse entre musulmans et chrétiens. Ce climat conflictuel dans les États voisins a toujours fait planer le spectre d'une révolte en provenance de l'islam majoritaire, une religion que le pouvoir politique tente de ce fait de maîtriser, de contrôler et de surveiller étroitement.

Le paradoxe actuel de la laïcité au Tchad naît du fait que, en un quart de siècle d'expérimentation démocratique, l'État tchadien qui s'est toujours voulu respectueux du principe de laïcité inscrit dans les différents textes

52. Charte déontologique, préambule. 
constitutionnels qui se sont succédé a fait preuve de beaucoup d'ambiguités. Le modèle de laiicité choisi, vidé de son sens originel de neutralité à la française, se trouve très souvent pris en otage par une pratique contournée. Le principe et sa mise en œuvre ont davantage servi les stratégies personnelles des dirigeants politiques, soucieux d'asseoir leur pouvoir. À titre d'exemple, on peut citer l'implication de l'État dans l'organisation du pèlerinage musulman. En effet, depuis 2015, le ministère de l'Intérieur a pris en main la gestion du hadj à La Mecque. La communauté musulmane n'a pas d'ailleurs manqué, à l'occasion de ses présentations des vœux au chef de l'État, de solliciter le maintien de cette implication étatique dans l'organisation du pèlerinage. Pour Cheikh Ahmat Annour Mahamat, imam de la mosquée centrale de N'Djamena, il en va du prestige de la nation de perpétuer cette action car «la Mecque est un lieu de rencontre mondiale qui fait que l'État ne peut pas se désintéresser de l'organisation du pèlerinage. Les pèlerins deviennent des ambassadeurs de leur pays ${ }^{53}$ ». Toujours dans ce même registre, on peut évoquer également les activités sociales des communautés musulmanes qui sont fortement appuyées et financées par les autorités politiques, de la base au sommet de l'État.

Cette approche paradoxale de la laïcité depuis la promulgation de la nouvelle constitution qui n'a pas manqué de faire l'objet de débats dans les milieux politiques, juridiques et médiatiques, s'inscrit encore dans cette confusion du modèle de laïcité qui est appliqué. L'introduction du serment confessionnel est l'idée d'un chef d'État qui outrepasse le cadre constitutionnel de neutralité pour s'immiscer dans le «salut des âmes ${ }^{54} »$ et, par là, s'attirer la sympathie d'une religion particulière, ici l'islam majoritaire. La construction d'un État démocratique, depuis la conférence nationale souveraine, a très vite été confrontée à des luttes intestines pour le partage du pouvoir et des richesses pétrolières dont le sous-sol tchadien regorge, amenant ainsi les dirigeants politiques successifs à chercher des alliances auprès des différentes communautés musulmanes, mais sans en officialiser la démarche comme dans le cas actuel. Le désenchantement des populations, confrontées à la précarité et qui s'impatientent de recevoir des bénéfices de la manne pétrolière, a conduit le pays à ne plus se reconnaître dans ses

53. Présentation des vœux du CSAIT au chef de l'État: https://presidence.td/fr-news-739. html [consulté le 24 avr. 2019].

54. Expression empruntée à John Locke: Lettre sur la tolérance, Londres, 1686, trad. par J. LeClerC, Paris, Flammarion, 1992, p. 19. Pour cet auteur, l'État ne doit pas s'occuper du salut des âmes. 
dirigeants. Dès lors, la question de la nécessité d'impliquer le religieux dans la gestion quotidienne de l'État s'est posée progressivement.

La construction d'un État véritablement laique voulu par le constituant tchadien depuis le processus démocratique est ainsi mise à mal par cette frontière perméable entre le politique et le religieux et peut, en un court laps de temps, conduire à une situation explosive. Le Tchad a encore du chemin à parcourir pour posséder une culture véritablement laïque qui permettrait une autonomisation respective du spirituel et du temporel ainsi qu'une prise en compte de la pluralité religieuse et de la diversité tant culturelle que cultuelle du pays. Face à cette imbrication du politique et du religieux, la laïcité tchadienne se métamorphose et permet désormais à l'État de raviver la sphère politique avec des dynamiques religieuses. De même, les institutions religieuses, musulmanes essentiellement, profitent de cette absorption dans le champ politique pour s'affirmer. Cette situation pourrait ne pas être sans danger pour la stabilité de cette partie de l'Afrique en proie aux conflits religieux. Par conséquent, il convient de résoudre la question de la laïcité en Afrique en évitant des arrangements et contradictions constitutionnels et en repensant un modèle de rapports religions-État compatible avec la société tchadienne, à l'aune de mutations politiques et religieuses où la nécessité d'une stabilité politique et d'un équilibre social prend de plus en plus le pas sur la démocratie et sur le modèle de laïcité-séparation traditionnellement consacré par le constituant. 